\title{
Estratégias de gestão de pessoas e desempenho organizacional na hotelaria: o papel das capacidades organizacionais
}

\author{
Human resource strategies and organizational performance in the hotel \\ sector: the role of organizational capabilities
}

\section{Estrategias de gestión de personas y desempeño organizacional en el sector hotelero: el papel de las capacidades organizacionales}

\author{
Leilianne Michelle Trindade da Silva Barreto ${ }^{1}$ \\ Lindolfo Galvão de Albuquerque ${ }^{2}$ \\ Carlos Alberto Freire Medeiros ${ }^{3}$
}

\begin{abstract}
Resumo: O objetivo geral desta pesquisa foi estabelecer relações entre configurações de estratégias de gestão de pessoas, capacidades organizacionais e desempenho organizacional na hotelaria. Foi desenvolvido um estudo descritivo e relacional, de abordagem quantitativa, nos hotéis dos principais destinos turísticos de quatro estados do Nordeste do Brasil. As relações encontradas confirmam a sobreposição proposta por Cameron e Quinn (2006) entre as estratégias de gestão de pessoas do Modelo de Valores Competitivos e as capacidades organizacionais do Modelo de Múltiplos Papéis de Ulrich (1998). Análises complementares atestaram que os hotéis que conseguem desenvolver as quatro capacidades organizacionais de forma equilibrada alcançam um desempenho superior.
\end{abstract}

Palavras-chave: Gestão Estratégica de Pessoas; Estratégias de Gestão de Pessoas; Capacidades Organizacionais; Desempenho Organizacional; Hotelaria.

\begin{abstract}
The general objective of this research was to establish relationships among the settings of human resource strategies, organizational capabilities and organizational performance in the hotel sector. A descriptive and relational study, with a quantitative approach, was developed in hotels of the principal tourist destinations of four states in the Northeast Region of Brazil. The relationships discovered confirm the super positioning proposed by Cameron and Quinn (2006) between the human resource strategies of the Competing Values Model and the organizational capabilities of Ulrich's (1998) Multiple Roles Model. Complementary analyses present testimony that hotels that are able to develop the four organizational capabilities in a equilibrated form, manage to reach higher performance.
\end{abstract}

1 Professora do Departamento de Turismo da Universidade Federal do Rio Grande do Norte (UFRN). Doutora em Administração pela Faculdade de Economia, Administração e Contabilidade da Universidade de São Paulo (FEA/USP). Mestre em Administração e Bacharel em Turismo pela UFRN. E-mail: leiliannebarreto@hotmail.com

2 Professor Titular da FEA/USP. Doutor, Mestre e Bacharel em Administração pela USP. E-mail: Igdalbuq@usp.br

3 Professor do Departamento de Administração da UFRN. Doutor em Administração pela FEA/USP. Mestre e Bacharel em Administração pela UFRN. E-mail: carlosalbertofreiremedeiros@gmail.com 
Keywords: Strategic Human Resource Management; Human Resource Strategies; Organizational Capabilities; Organizational Performance; Hotel Sector.

Resumen: El objetivo general de esta investigación fue establecer las relaciones entre las configuraciones de estrategias de gestión de personas, las capacidades organizacionales y el desempeño organizacional en el sector hotelero. Fue desarrollado un estudio descriptivo y relacional, con enfoque cuantitativo, en los hoteles de los principales destinos turísticos en cuatro estados en el noreste de Brasil. Las relaciones encontradas confirman la superposición propuesto por Cameron y Quinn (2006) entre las estrategias de gestión de personas del Modelo de Valores Competitivos e las capacidades organizacionales del Modelo de Roles Múltiples de Ulrich (1998). Un análisis más detallado testificó que los hoteles que se pueden desarrollar las cuatro capacidades organizacionales de manera uniforme alcanzan el máximo rendimiento.

Palabras clave: Gestión Estratégica de Personas; Estrategias de Gestión de Personas; Capacidades Organizacionales; Desempeño Organizacional; Sector Hotelero.

\section{INTRODUÇÃO}

A gestão estratégica de pessoas (GEP) é um tema que tem recebido crescente atenção nos últimos anos. É possível afirmar que o foco principal dos trabalhos nessa área é a busca pela vantagem competitiva das organizações. De acordo com Ulrich (2000), as questões sobre como criar valor nas organizações sempre permearam as discussões organizacionais, configurando-se em desafios para os líderes. A diferença presente no contexto dos últimos anos é que a gestão de pessoas tem ganhado espaço crescente na tentativa de responder a esses desafios, assumindo um papel cada vez mais estratégico e orientado para os resultados.

Assim, várias pesquisas são realizadas no intuito de investigar as associações entre as políticas e práticas de gestão de pessoas e o desempenho organizacional (Becker, Huselid, \& Ulrich, 2001; Martín-Alcázar, Romero-Fernández, \& Sánchez-Gardey, 2008; Deadrick \& Gibson, 2009; Lengnick-Hall, Lengnick-Hall, Andrade, \& Drake, 2009; Ketkar \& Sett, 2010; Wei \& Lau, 2010; McClean \& Collins, 2011. Nessa mesma linha, também é numerosa a produção que destaca a necessidade do alinhamento estratégico para o alcance de vantagem competitiva sustentável, abordando a integração vertical entre as estratégias de negócios e as estratégias de gestão de pessoas (Schuler \& Jackson, 1987; Miles \& Snow, 1984; Wright, McMahan, \& McWilliams, 1994; Barney \& Wright, 1998; Panayotopoulou, Bourantas, \& Papalexandris, 2003; Jin, Hopkins, \& Wittmer, 2010)

Estudos recentes têm reforçado a importância de analisar os fatores de implementação de estratégias e a necessidade de mensurar as práticas de gestão de pessoas realizadas, avaliando seus respectivos resultados (Lengnick-Hall, Lengnick-Hall, Andrade, \& Drake, 2009). Isso conduz ao crescente interesse na área de avaliação de resultados provenientes da gestão de pessoas, suscitando a mensuração da contribuição desta para os resultados organizacionais, como uma etapa importante do ciclo de gestão da estratégia.

Para Lacombe e Albuquerque (2008), o tema de avaliação de resultados em gestão de pessoas ganhou relevância especial porque o seu delineamento estratégico passou a demandar 
um maior volume de investimento em pessoas, o qual precisa ser justificado através do retorno que ele traz para a organização. Porém, os autores destacam que ainda existe pouca pesquisa a respeito do tema no contexto brasileiro.

Nessa retórica de busca por resultados, tão marcante entre as organizações que se deparam com um mercado cada vez mais competitivo, muito já se pesquisou acerca da constatação de associações entre as políticas e práticas de gestão de pessoas e o desempenho organizacional e até mesmo a respeito dos motivos dessas associações, mas pouco se investigou sobre como a gestão de pessoas leva aos resultados organizacionais desejados.

Reafirmando essa lacuna, Ulrich $(2000 ; 1998)$ comenta que vários estudos comprovam relações positivas entre as práticas de gestão de pessoas e/ou investimentos na área e os resultados financeiros das organizações. Porém, poucas são as explicações sobre o motivo da existência dessas relações e sobre a maneira como elas acontecem. Logo, torna-se importante examinar as etapas intermediárias desse processo, descrevendo o caminho percorrido entre a gestão de pessoas e os resultados organizacionais. Para o autor, uma maneira interessante para começar a desvendar a causa dessas associações é a avaliação dos resultados de gestão de pessoas definidos em termos de capacidades organizacionais.

Vários pesquisadores também têm chamado a atenção especialmente para estudos que endereçam questões de investigação no nível intermediário, a fim de revelar os fatores responsáveis pela mediação entre a gestão estratégica de pessoas e o desempenho organizacional (Ketkar \& Sett, 2010; McClean \& Collins, 2011).

Segundo Legge (2005), pouco tem sido feito para destravar a "caixa preta" do processo que relaciona gestão de pessoas a desempenho organizacional. Ela advoga que o caminho para isso é o desenvolvimento de modelos que incluam variáveis intervenientes chave. Entretanto, a maioria dos estudos que se propõe a inserir variáveis mediadoras na relação GEP-desempenho emprega fatores individuais em seus modelos conceituais, geralmente associados ao comportamento e desempenho dos funcionários. Poucos são os estudos que inserem algum fator no nível organizacional para desvendar o processo de geração de resultados da gestão estratégica de pessoas.

Nesse nível de análise, Roehling et al. (2005) e Wei e Lau (2010) - assim como Ulrich (2000; 1998) - também sugerem que as capacidades organizacionais mediam tal relação, uma vez que elas são percebidas como resultados da gestão de pessoas. Entretanto, eles afirmam que ainda se conhece muito pouco sobre como a gestão de pessoas afeta o desenvolvimento e a manutenção das capacidades organizacionais e que ainda é preciso investigar as relações entre estas e a efetividade organizacional.

Nesse contexto, o presente trabalho se propõe a estabelecer relações entre as configurações de estratégias de gestão de pessoas, as capacidades organizacionais e o desempenho organizacional dos hotéis do Nordeste do Brasil, oferecendo subsídios para a gestão eficaz dos hotéis e para a maximização de seus resultados.

Para fundamentar essa discussão, inicialmente serão apresentadas as bases teóricas 
relativas aos constructos e os respectivos modelos selecionados para utilização no estudo empírico. Os tópicos seguintes apresentam os aspectos metodológicos da pesquisa de campo e a discussão dos resultados, encerrando com as considerações finais.

\section{ESTRATÉGIAS DE GESTÃO DE PESSOAS}

Schuler e Walker (1990) elaboraram uma das primeiras definições de estratégias de gestão de pessoas. Para estes autores, elas podem ser concebidas como um processo e um conjunto de atividades que identificam questões específicas de gestão de pessoas que são de interesse do negócio. Essa atividade é baseada em um esforço conjunto dos recursos humanos e dos gerentes de linha em responder às preocupações do negócio relacionadas às pessoas, visando à consecução dos objetivos estratégicos do negócio e agregando valor à organização, para melhorar o seu desempenho atual e futuro e sustentar a vantagem competitiva.

Albuquerque (1999) transformou-se num clássico da literatura brasileira sobre estratégias de gestão de pessoas. Ele explorou e aprimorou os conceitos de duas estratégias básicas de gestão de pessoas: a estratégia de controle e a estratégia de comprometimento. A primeira é caracterizada por uma estrutura altamente hierarquizada, com produção em massa, trabalho especializado, com baixo nível de exigência em qualificação e baixa participação dos empregados na tomada de decisões. Já a segunda é caracterizada pela produção flexível, com a junção entre o fazer e o pensar, gerando trabalhos enriquecidos e desafiadores, alta exigência de qualificação, confiança mútua e alta participação dos empregados nas tomadas de decisões.

De forma similar, Truss, Gratton, Hope-Hailey, McGovern e Stiles (1997), baseados em outros autores, trabalharam essas estratégias como modelos hard e soft. O modelo hard é equivalente à estratégia de controle, baseado no modelo econômico do homem da teoria $X$ de McGregor, evidenciando aspectos quantitativos, racionais, e enfatizando a ideia de recursos. Já o modelo soft está associado ao movimento das relações humanas, à utilização dos talentos individuais e à teoria $Y$ de McGregor sobre a natureza dos indivíduos, o que equivaleria à estratégia de comprometimento, a qual é baseada em altos níveis de confiança. Em sua pesquisa empírica em organizações na Inglaterra, os autores concluíram que o modelo soft de comprometimento é predominante na retórica adotada pelas empresas. Entretanto, a prática vivenciada pelos empregados está mais relacionada ao modelo hard, de controle estratégico.

Para Mascarenhas (2008), dependendo das demandas estratégicas das organizações, seriam compatíveis ênfases diferentes dos modelos soft e hard. O modelo soft, por exemplo, seria mais adequado para organizações inseridas em cenários instáveis, que exigem estruturas mais flexíveis e maior envolvimento dos funcionários em processos estratégicos de mudança e inovação. Já o modelo hard seria mais adequado para cenários estáveis, que enfatizam a produtividade, a continuidade de processos e comportamentos repetitivos. O autor considera ainda a possibilidade da coexistência das duas abordagens, para prover tratamentos diferenciados aos subgrupos organizacionais. 
Com uma visão crítica, Legge (2005) afirma que existia uma tendência no processo de alinhamento entre gestão de pessoas e estratégia organizacional em escolher entre uma estratégia de gestão de pessoas hard ou soft, dependendo de a organização possuir uma estratégia de liderança no custo ou de alto valor agregado. Entretanto, reconhece-se atualmente que não é mais uma questão de escolher "um dos dois" ou "ou uma" estratégia, mas sim de "ambos" ou "e", incorporando mais de uma estratégia à gestão de pessoas.

Um modelo que incorpora a visão de Legge (2005) é o Modelo de Valores Competitivos (Competing Values Framework - CVF), que tem sido usado para definir diferentes abordagens da gestão de pessoas nas organizações. Ele foi desenvolvido por Quinn e Rohrbaugh (1983) como um modelo para a análise organizacional, sendo bastante amplo e aplicável à investigação de diversos aspectos, tais como: cultura organizacional, liderança, efetividade organizacional, gestão da qualidade e gestão de pessoas, conforme sugerido por Cameron e Quinn (2006).

O modelo está fundamentado em quatro conjuntos de valores que competem entre si, refletindo os dilemas que permeiam o sistema organizacional. Ressalta-se que ele não sugere que um valor anula o outro; ao contrário, tenta proporcionar uma melhor compreensão do equilíbrio entre eles. O modelo compreende uma combinação de duas dimensões: a) a primeira está baseada na estrutura organizacional, com possibilidade de ênfase em flexibilidade e dinamismo ou em estabilidade, ordem e controle; b) a segunda dimensão está relacionada ao enfoque organizacional, diferenciando a ênfase na orientação para o ambiente interno, integração e unidade, da orientação para o ambiente externo, diferenciação e rivalidade. Essas duas dimensões dão origem a quatro quadrantes, que representam modelos distintos, de acordo com as principais características e valores assumidos (Figura 1).

O modelo de relações humanas é caracterizado pela flexibilidade e foco interno. Ele enfatiza trabalho em equipe e comprometimento dos funcionários através do desenvolvimento de um forte sistema de valores que promove a identidade corporativa. As boas relações entre os funcionários são um resultado da meritocracia, oportunidades iguais, participação e envolvimento. A motivação é baseada no empoderamento, desenvolvimento e comunicação, enquanto o sucesso é definido em termos da valorização das pessoas.

O modelo sistema aberto é definido pela flexibilidade e foco externo. A cultura dominante fomenta a inovação, a criatividade, o empreendedorismo e a tomada de riscos e objetiva a criação de uma visão do futuro da organização. A principal responsabilidade do RH é acompanhar as mudanças ambientais, sendo capaz de se adaptar a elas através de melhoria contínua, aquisição de novos recursos e adoção de novos métodos e processos. O sucesso é definido em função da flexibilidade e adaptabilidade à mudança.

O modelo de processo interno é caracterizado pelo controle e foco interno. Enfatiza o controle próximo dos funcionários, a padronização de procedimentos, a gestão da informação e a manutenção da estabilidade e da hierarquia. A melhoria de processos, regulamentos e análise do trabalho, através de métodos como a reengenharia, facilita o trabalho do RH. Previsibilidade e eficiência do processo são critérios de sucesso. 
Figura 1 - Modelo de valores competitivos para gestão de pessoas

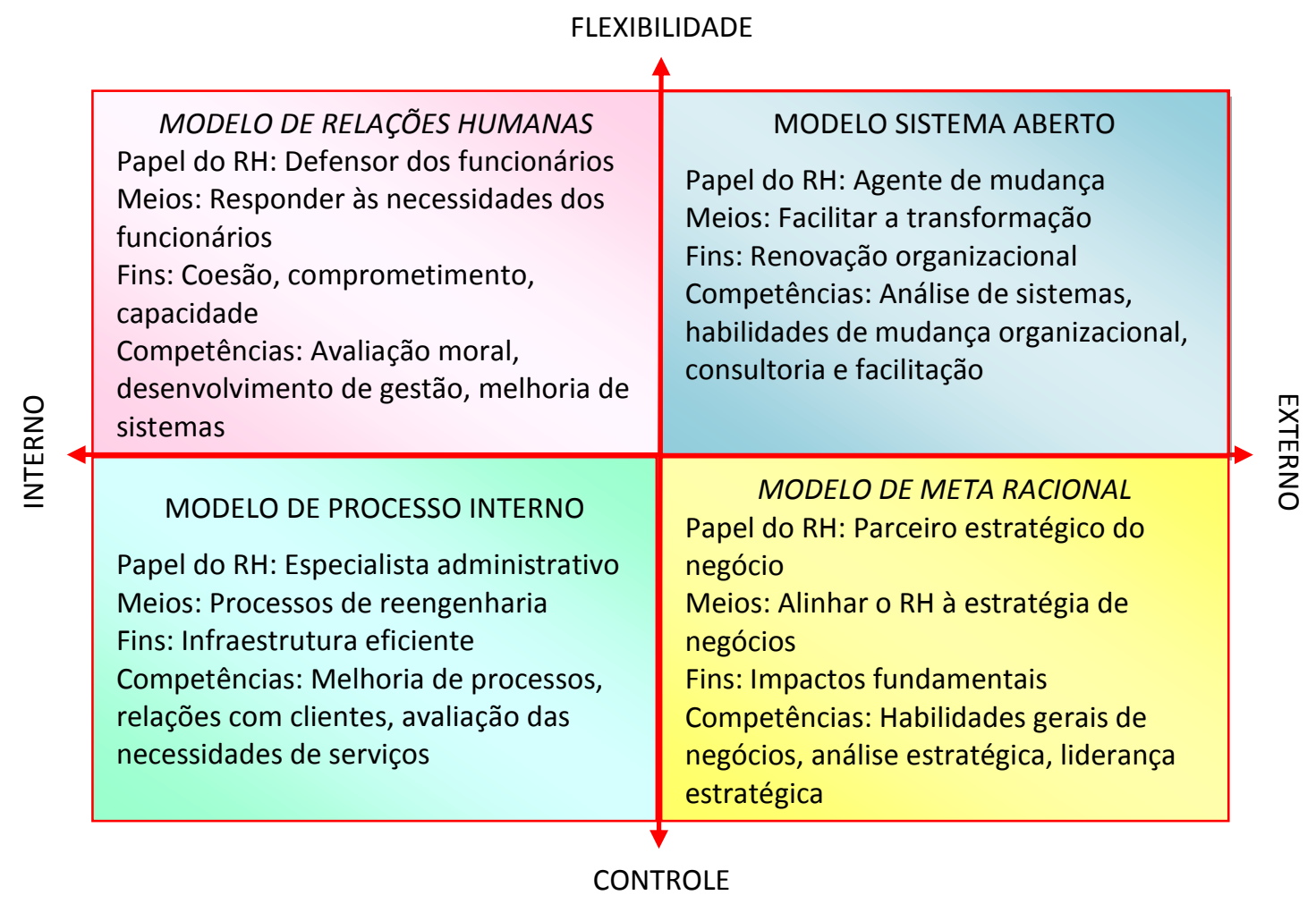

Fonte: Adaptado de Cameron \& Quinn (2006)

Já o modelo de meta racional é evidenciado pelo controle e foco externo. Sua característica básica é a orientação para resultados. Para tanto, o RH enfatiza planejamento, conquista de metas, medidas de produtividade e competitividade. Seu principal papel é ser um parceiro estratégico, através do alinhamento de suas políticas com a estratégia de negócios. Rentabilidade, eficiência, produtividade, fama e competitividade são critérios de sucesso.

Vale destacar que o Modelo de Valores Competitivos servirá como base para as análises realizadas neste trabalho. Cameron e Quinn (2006) delineiam o seu emprego para a gestão de pessoas, propondo uma sobreposição com o Modelo de Múltiplos Papéis da gestão de pessoas de Ulrich (1998), o qual é detalhado no tópico seguinte.

\section{CAPACIDADES ORGANIZACIONAIS COMO RESULTADOS DA GESTÃO DE PESSOAS}

Com o advento da perspectiva estratégica, a gestão de pessoas tem sofrido uma mudança de foco, migrando de uma ênfase baseada em processos e tarefas para uma nova ênfase mais proativa, baseada em resultados.

Para Oliveira, Albuquerque e Muritiba (2003), quanto mais os gestores confirmarem a contribuição da gestão de pessoas para a organização, mais a área será percebida como uma parceira estratégica, ao mesmo tempo em que a maior participação da área na gestão estratégica 
implica maior demanda pela avaliação de resultados. Logo, seja para alcançar ou para manter o status estratégico, a avaliação de resultados torna-se imperativa para a gestão de pessoas, impondo aos seus gestores os desafios sobre a definição do que medir e a tradução dos indicadores em informações relevantes para os negócios.

Lacombe e Albuquerque (2008, p. 8) comentam que os autores que propõem métodos de mensuração de resultados em gestão de pessoas parecem concordar que existem dois grandes tipos de medidas para a área: as medidas operacionais - tais como índices de absenteísmo, rotatividade, horas de treinamento etc. - e os indicadores estratégicos. Estes procuram "avaliar não apenas as ações já passadas, mas também como as ações de RH podem influenciar o futuro da organização, especialmente em termos da construção das capacidades organizacionais". De modo similar, Ulrich $(2000 ; 1998)$ também defende que as capacidades organizacionais se constituem em importantes resultados da gestão de pessoas - premissa adotada neste estudo.

Segundo Mascarenhas (2008), o reconhecimento de que a gestão de pessoas desempenha uma função importante na construção de capacidades organizacionais deve-se ao fato de ela estar por trás da mobilização e da reconfiguração dos recursos competitivos. Logo, as capacidades organizacionais são resultados da gestão de pessoas críticos para o alcance de desempenho organizacional superior (Ulrich \& Smallwood, 2005).

Baseados na perspectiva das capacidades dinâmicas, Wei e Lau (2010) confirmaram que a relação entre gestão de pessoas e desempenho organizacional é parcialmente mediada pela capacidade adaptativa das empresas. Resultados semelhantes foram encontrados por Jin, Hopkins e Wittmer (2010), que verificaram que a flexibilidade das empresas, definida como a capacidade de responder com eficiência e eficácia às exigências dos clientes, media a relação entre o seu capital humano e as suas vantagens competitivas.

Ulrich $(2000 ; 1998)$ advoga que as políticas e práticas de gestão de pessoas devem ser desenvolvidas de forma integrada para criar capacidades que contribuam para o sucesso organizacional. Em sua proposição de valor, o autor defende que as práticas de gestão de pessoas criam capacidades organizacionais que irão gerar valor para o cliente, o que, por sua vez, irá se converter em valor econômico. Logo, as capacidades organizacionais representam o elo entre estratégia e ação e entre a gestão de pessoas e o desempenho organizacional, sendo responsabilidade da gestão de pessoas a transformação de estratégias em capacidades.

De acordo com o autor, existem quatro capacidades genéricas essenciais que podem ser definidas como resultados da gestão de pessoas: criar clareza estratégica, fazer com que as mudanças aconteçam, gerar capital intelectual e criar eficiência administrativa. Essas capacidades derivam de seu Modelo de Múltiplos Papéis, apresentado na Figura 2, que advoga que esses papéis devem ser assumidos pelos profissionais de gestão de pessoas concomitantemente, para que eles possam adicionar valor às organizações competitivas. 
Figura 2 - Papéis de RH na construção de uma organização competitiva.

FUTURO/ESTRATÉGICO

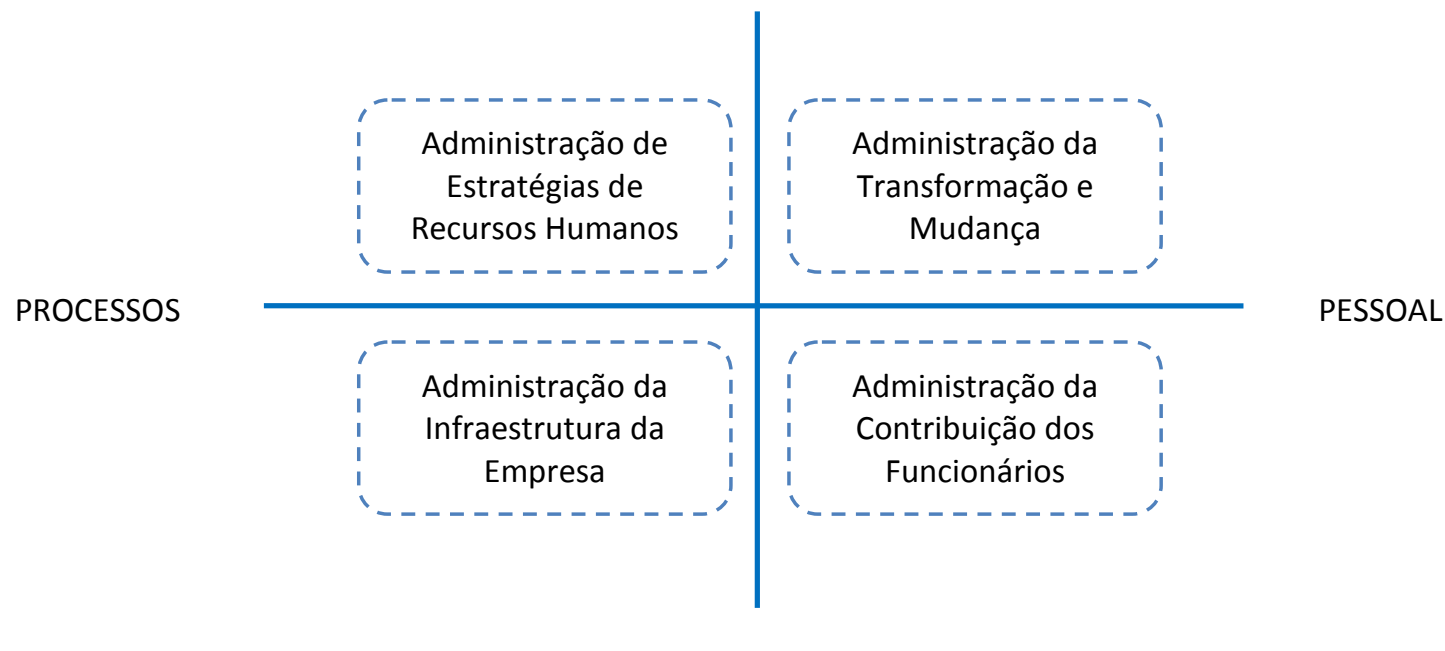

COTIDIANO/OPERACIONAL

Fonte: Ulrich (1998, p. 40)

O modelo trabalha com duas dimensões: o foco e as atividades de gestão de pessoas. 0 foco pode variar entre o estratégico, orientado para o futuro, e o operacional, voltado para o cotidiano. As atividades vão da administração de processos à administração de pessoal. Os quadrantes delineados no cruzamento dessas duas dimensões dão origem a quatro papéis de gestão de pessoas: a administração de estratégias de recursos humanos, que resultaria na capacidade de criar clareza estratégica; a administração da transformação e mudança, cujo resultado correspondente seria a capacidade de fazer com que as mudanças aconteçam; a administração da contribuição dos funcionários, que levaria à capacidade de gerar capital intelectual; e a administração da infraestrutura da empresa, que teria como resultado a capacidade de criar eficiência administrativa.

A capacidade de criar clareza estratégica propicia o alcance dos objetivos organizacionais e ajuda a garantir o sucesso, gerando significado para os seus funcionários e para os demais stakeholders. Ela alinha os sistemas de gestão de pessoas às estratégias empresariais, traduzindoas em práticas organizacionais de forma eficiente, moldando o comportamento dos funcionários e diferenciando a empresa para clientes e investidores.

A capacidade de fazer com que as mudanças aconteçam proporciona flexibilidade, velocidade e agilidade às organizações no processo de rápida adaptação ao ambiente competitivo dinâmico e instável. Ela facilita a inovação e a adaptação constantes, contribuindo para a compreensão dos processos cruciais para a mudança, gerando dedicação a esses processos e garantindo que ela ocorra de acordo com o planejado.

A capacidade de gerar capital intelectual favorece maior comprometimento e competência dos funcionários. As práticas de gestão de pessoas ajudam os funcionários a entregar resultados - 
através de suas competências individuais - que contribuam para o sucesso organizacional. Isso ocorre a partir da compreensão das necessidades dos funcionários, elevando sua contribuição e seu comprometimento com as metas da empresa.

A capacidade de criar eficiência administrativa facilita a concepção e desenvolvimento de processos de maneira eficiente. Ela envolve a melhoria contínua dos processos de gestão de pessoas e o aumento da eficiência geral da organização, na busca incessante por descobrir maneiras novas de fazer as coisas melhor, com o intuito de garantir a manutenção da qualidade e a redução dos custos.

Para Ulrich (2000, p. 39), essas quatro capacidades são complementares e devem ser desenvolvidas em conjunto para desempenhar uma parceria ampla e global que de fato possa agregar valor às organizações, como ilustra o trecho a seguir: "Empresas bem-sucedidas serão aquelas capazes de transformar estratégia em ação rapidamente, de gerenciar processos de maneira inteligente e eficiente, de maximizar o compromisso e a colaboração do funcionário e de criar condições para uma mudança consistente".

Vale ressaltar que esse modelo de quatro capacidades organizacionais como resultados da gestão de pessoas será adotado para a realização do estudo empírico, conforme descrito na metodologia da pesquisa.

\section{ASPECTOS METODOLÓGICOS}

Este estudo se caracteriza como descritivo e relacional, uma vez que se propôs a estabelecer relações entre as estratégias de gestão de pessoas, as capacidades organizacionais e o desempenho organizacional dos hotéis do Nordeste brasileiro. Em relação à abordagem metodológica, este estudo caracteriza-se como um levantamento (survey), fazendo uso de métodos quantitativos.

\subsection{População e Amostra}

A população desta pesquisa abrange os hotéis localizados nos dois principais destinos turísticos de quatro estados do Nordeste do Brasil, a saber: Salvador/BA, Porto Seguro/BA, Fortaleza/CE, Canoa Quebrada/CE, Recife/PE, Porto de Galinhas/PE, Natal/RN e Pipa/RN. Em virtude dos objetivos da pesquisa, foram selecionados para compor a amostra apenas os hotéis que possuem o mínimo de 30 unidades habitacionais.

O arcabouço amostral era de 250 hotéis. Alguns deles se recusaram a participar da pesquisa e outros não puderam responder durante o período de coleta de dados, alegando como justificativa a limitação de tempo. Desse modo, a amostra constituiu-se como não probabilística, por conveniência, obtendo-se uma amostra válida de 151 hotéis, o que representa uma taxa de retorno de $60,40 \%$. 


\subsection{Coleta de Dados}

Os dados primários foram coletados através de uma pesquisa de campo in loco, com aplicação de questionários aos gestores dos meios de hospedagem.

$\mathrm{O}$ instrumento de coleta de dados é composto por cinco partes. A primeira parte engloba as estratégias de gestão de pessoas. Elas foram avaliadas segundo o Modelo de Valores Competitivos (Quinn \& Rohrbaugh, 1983; Cameron \& Quinn, 2006) para mensurar as quatro configurações de gestão de pessoas: Meta Racional, Sistema Aberto, Relações Humanas e Processo Interno.

A segunda parte do questionário investiga as capacidades organizacionais com base no Modelo de Múltiplos Papéis de Ulrich (1998; 2000). Com esta parte foram mensuradas as quatro capacidades organizacionais genéricas: Clareza Estratégica, Mudança, Capital Intelectual e Eficiência Administrativa.

$\mathrm{Na}$ terceira parte do questionário foram coletados dados acerca do desempenho organizacional, que foi pesquisado a partir de cinco indicadores: taxa de ocupação hoteleira, valor da diária média, desempenho financeiro, produtividade do trabalho e qualidade dos serviços. A quarta e a quinta partes abordam informações gerais sobre os meios de hospedagem e seus respondentes, respectivamente. Todos os indicadores do questionário foram mensurados com o uso de escalas de percepção que variam de 0 a 10.

\subsection{Análise de Dados}

Os dados foram tabulados, mensurados e analisados de forma quantitativa. Inicialmente, as escalas de mensuração dos três construtos centrais da pesquisa (estratégias de gestão de pessoas, capacidades organizacionais e desempenho organizacional), foram refinadas com 0 auxílio das técnicas de análise fatorial e análise de confiabilidade. A análise fatorial foi utilizada para confirmar as dimensões teóricas e o agrupamento dos indicadores, condensando as informações e resumindo os dados para facilitar a aplicação das análises relacionais posteriores. Já a análise de confiabilidade permitiu atestar a consistência interna dos indicadores utilizados para compor as dimensões teóricas utilizadas, gerando coeficientes alfa de Cronbach bastante elevados, todos superiores a 0,85 .

Num segundo momento, as relações entre as dimensões dos construtos em questão foram analisadas através do uso das seguintes técnicas estatísticas: modelagem de equações estruturais, análise de regressão múltipla, análise de conglomerados e teste t de Student. A aplicação de cada uma das técnicas está explicitada ao longo da descrição dos resultados da pesquisa. 


\section{RELAÇÕES ENTRE ESTRATÉGIAS DE GESTÃO DE PESSOAS, CAPACIDADES ORGANIZACIONAIS E DESEMPENHO ORGANIZACIONAL}

Com base nos pressupostos teóricos do Modelo de Valores Competitivos (Cameron \& Quinn, 2006) e do Modelo de Múltiplos Papéis de Ulrich (2000), procedeu-se ao uso da técnica de modelagem de equações estruturais para investigar as relações simultâneas entre estratégias de gestão de pessoas, capacidades organizacionais e desempenho organizacional dos meios de hospedagem. O primeiro passo na modelagem de equações estruturais é a realização da análise de caminhos, quando se definem as relações preditivas entre os construtos, com a definição das variáveis dependentes e independentes. Assim, para a análise de caminhos, consideraram-se como variáveis exógenas (independentes) as configurações de estratégias de gestão de pessoas e como variáveis endógenas (dependentes) as capacidades organizacionais e o desempenho organizacional. Tal análise foi realizada para verificar quais estratégias de gestão de pessoas implementadas pelos meios de hospedagem do Nordeste brasileiro serviam para explicar as capacidades organizacionais por eles desenvolvidas e, ainda, para identificar quais destas capacidades contribuem para explicar as variações de desempenho organizacional entre os hotéis investigados.

A Figura 3 ilustra o modelo obtido a partir da análise de caminhos. A avaliação do ajuste do modelo revela que ele possui um bom ajuste. A significância do teste qui-quadrado foi igual a 0,000 , e o teste qui-quadrado normado, que é a razão entre a estatística qui-quadrado e os graus de liberdade, foi igual a 3,39. Esse valor é aceitável, tendo em vista que a literatura recomenda que o qui-quadrado normado seja de no máximo 5, para indicar um ajuste adequado. 
Figura 3 - Modelo Estratégias de Gestão de Pessoas - Capacidades Organizacionais - Desempenho Organizacional

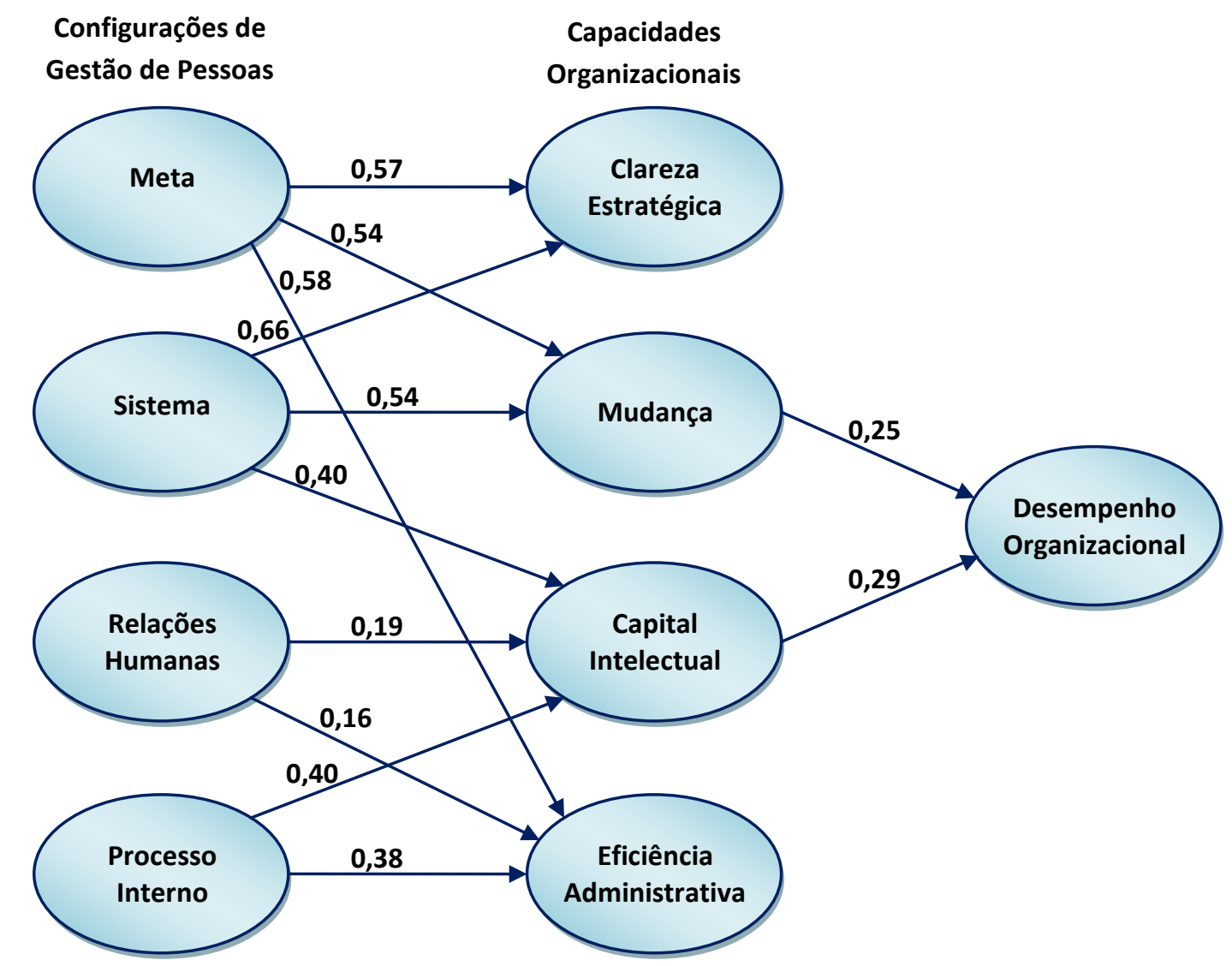

O lado esquerdo do modelo revela a influência das estratégias de gestão de pessoas sobre as capacidades organizacionais. A configuração Meta Racional, que tem forte orientação para resultados, conquista de metas, produtividade e competitividade, exerce nítida influência sobre as capacidades de Clareza Estratégica, Mudança e Eficiência Administrativa. A capacidade de Clareza Estratégica provavelmente recebe essa influência por compartilhar com a configuração Meta Racional a orientação para o ambiente externo, a visão estratégica, o controle e a administração de processos. A capacidade de Mudança compartilha a orientação para o ambiente externo e a visão estratégica. Já a capacidade de Eficiência Administrativa compartilha a ênfase sobre o controle e a administração de processos.

A configuração Sistema Aberto, que evidencia o senso de renovação, mudança, melhoria contínua, criatividade e iniciativas inovadoras, explica positivamente as capacidades de Clareza Estratégica, Mudança e Capital Intelectual. A capacidade de Clareza Estratégica recebe essa influência por compartilhar com a configuração Sistema Aberto a orientação para o ambiente externo e a visão estratégica. A capacidade de Mudança compartilha a orientação para o ambiente 
externo, a visão estratégica, a flexibilidade e a administração de pessoal. Já a capacidade de Capital Intelectual compartilha a flexibilidade e a administração de pessoal.

A configuração Relações Humanas, que possui ênfase sobre o trabalho em equipe, o comprometimento dos funcionários e as boas relações de trabalho, exerce influência positiva sobre as capacidades de Capital Intelectual e Eficiência Administrativa. A capacidade de Capital Intelectual recebe essa influência por compartilhar com a configuração Relações Humanas a orientação para o ambiente interno, o operacional, a flexibilidade e a administração de pessoal. A capacidade de Eficiência Administrativa compartilha a orientação para o ambiente interno e o operacional.

A configuração Processo Interno, que ressalta a eficiência e a melhoria dos processos e a gestão da informação, influencia positivamente as capacidades de Capital Intelectual e Eficiência Administrativa. A capacidade de Capital Intelectual recebe essa influência por compartilhar com a configuração Processo Interno a orientação para o ambiente interno e o cotidiano. A capacidade de Eficiência Administrativa compartilha a orientação para o ambiente interno, o cotidiano, o controle e a administração de processos.

Um aspecto a se destacar no modelo é que ele confirma a sobreposição proposta por Cameron e Quinn (2006) entre as estratégias de gestão de pessoas do Modelo de Valores Competitivos e as capacidades organizacionais do Modelo de Múltiplos Papéis de Ulrich (1998). A referida sobreposição indica que: a) a configuração Meta Racional, caracterizada pelo controle e foco externo, contribui para o desenvolvimento da capacidade de Clareza Estratégica, voltada para o futuro e para a administração de processos; b) a configuração Sistema Aberto, caracterizada pela flexibilidade e foco externo, contribui para o desenvolvimento da capacidade de Mudança, voltada para o futuro e para a administração de pessoal; c) a configuração Relações Humanas, caracterizada pela flexibilidade e foco interno, contribui para o desenvolvimento da capacidade de Capital Intelectual, voltada para o cotidiano e para a administração de pessoal; e d) a configuração Processo Interno, caracterizada pelo controle e foco interno, contribui para o desenvolvimento da capacidade de Eficiência Administrativa, voltada para o cotidiano e para a administração de processos.

Além disso, observa-se que as relações não se apresentam de forma isolada entre cada dupla de dimensões correspondentes. Ao contrário, as relações são múltiplas, ou seja, cada configuração de estratégias de gestão de pessoas influencia concomitantemente o desenvolvimento de duas ou mais capacidades organizacionais. Mais uma vez, a proposição de Cameron e Quinn (2006) é reforçada, quando eles afirmam que, para alcançar a efetividade, os gestores devem garantir que alguns elementos de cada um dos quatro modelos de gestão de pessoas sejam contemplados pelas organizações.

Essa observação também resgata a reflexão de que os tipos ideais identificados pelos estudiosos da abordagem configuracional da gestão estratégica de pessoas dificilmente serão percebidos na prática organizacional em sua forma "pura". O que ocorre, na realidade, são as composições de modelos híbridos, que combinam características de diversos elementos, sendo 
mais apropriados à natureza complexa e interativa da gestão de pessoas nas organizações (Delery \& Doty, 1996).

O lado direito do modelo revela a influência das capacidades organizacionais sobre o desempenho organizacional dos meios de hospedagem. Dentre as quatro capacidades organizacionais investigadas, apenas duas exercem influência positiva direta sobre o desempenho organizacional: Mudança e Capital Intelectual. A capacidade de Mudança envolve o favorecimento da inovação e da adaptação constante e o comprometimento com os processos de mudança. $O$ próprio Ulrich (2000) defende que, a partir do senso de melhoria contínua, tem-se o estímulo a novas iniciativas que asseguram a continuidade e o aumento do desempenho organizacional. Já a capacidade de Capital Intelectual ressalta a contribuição dos funcionários. Assim, Ulrich (2000) argumenta que, através das competências individuais dos funcionários e do seu comprometimento com as metas da organização, eles entregam resultados que contribuem para o sucesso organizacional. Esse resultado vem corroborar a visão de vários autores, que afirmam que os ativos mais valiosos de uma organização são os funcionários e que as pessoas possuem valor estratégico e representam fonte de vantagem competitiva para as organizações (Barney, 1991; Wright, Dunford, \& Snell, 2001).

Analisando-se a descrição teórica apresentada por Ulrich (2000; 1998) em seu Modelo de Múltiplos Papéis, percebe-se que ele dá maior ênfase às contribuições das capacidades de Mudança e de Capital Intelectual sobre o desempenho e o sucesso organizacional. Já a sua descrição sobre as capacidades de Clareza Estratégica e de Eficiência Administrativa evidencia a preparação da organização para o enfrentamento de desafios operacionais e estratégicos, mas não salienta o impacto direto sobre o desempenho organizacional. Essa observação poderia justificar o não aparecimento de relações significantes entre essas duas capacidades e o desempenho organizacional.

Ao mesmo tempo, vale ressaltar que as capacidades de Mudança e de Capital Intelectual estão ambas voltadas para as atividades de administração de pessoal, ou seja, possuem maior ênfase sobre as pessoas e traduzem maior flexibilidade, seja evidenciando a inovação e a melhoria contínua (Mudança), seja apontando o comprometimento e as competências dos funcionários (Capital Intelectual). O fato de elas terem se sobressaído na influência sobre o desempenho organizacional dos meios de hospedagem é coerente com os resultados de outras pesquisas desenvolvidas em consonância com a teoria das capacidades dinâmicas, cujos autores constataram que o desenvolvimento de capacidades organizacionais que facilitam a transformação e a rápida adaptação às mudanças do ambiente competitivo e que encorajam e capacitam seus funcionários são mediadoras das relações entre gestão de pessoas e desempenho organizacional (Wei \& Lau, 2010; Jin, Hopkins, \& Wittmer, 2010; Lengnick-Hall, Beck, \& LengnickHall, 2011).

Entretanto, Ulrich (2000) defende que as quatro capacidades são complementares e devem ser desenvolvidas em conjunto para proporcionar diferencial competitivo às organizações, sugerindo assim uma ideia de equilíbrio entre as capacidades organizacionais. Logo, esperava-se 
que as capacidades de Clareza Estratégica, que ressalta o alinhamento entre os sistemas de gestão de pessoas e as estratégias empresariais, e de Eficiência Administrativa, que enfatiza a eficiência e a melhoria contínua dos processos, também apresentassem influência positiva direta sobre o desempenho organizacional - embora de maneira mais fraca que as demais - o que não se confirmou. Isso pode ter acontecido devido à variação das respostas referentes aos indicadores que compõem essas duas dimensões ter sido mais baixa que a variação das respostas relativas às outras duas dimensões que se apresentaram como determinantes significantes do desempenho organizacional. Essa constatação sugere que as capacidades de Clareza Estratégica e de Eficiência Administrativa não contribuem tanto quanto as outras para diferenciar os hotéis pesquisados.

O grau de desenvolvimento das capacidades organizacionais apontado pelos gestores dos meios de hospedagem pesquisados revelam que o valor médio mais baixo foi atribuído à capacidade de Clareza Estratégica e o valor médio mais alto foi conferido à capacidade de Eficiência Administrativa, o que revelou uma maior dificuldade de desenvolver atividades estratégicas e orientadas para o futuro e uma maior facilidade de desenvolver atividades operacionais e orientadas para o cotidiano. Assim, acredita-se que essas duas capacidades não foram contempladas no modelo como determinantes do desempenho porque a maioria dos hotéis, apesar de apresentarem diferenças consideráveis de desempenho organizacional entre si, não se diferenciam muito em relação a essas duas capacidades, pois suas dificuldades e facilidades são semelhantes.

Essa constatação demonstra que os empreendimentos hoteleiros estão explorando pouco a possibilidade de serem diferentes, especialmente no que concerne às suas estratégias organizacionais. Como afirmam os teóricos da RBV (Penrose, 1959; Barney, 1991; Teece, Pisano, \& Shuen, 1997), é a heterogeneidade dos recursos e das capacidades que conduzem as organizações a patamares distintos de resultados. Logo, os hotéis que minimizarem a lacuna que ainda persiste para o desenvolvimento da capacidade de Clareza Estratégica, possivelmente irão oferecer um diferencial competitivo aos clientes e alcançar um bom posicionamento de mercado.

Pelo fato de a variável desempenho organizacional não ter apresentado todas as relações esperadas, tentou-se verificar como as capacidades organizacionais se relacionam com cada um dos cinco indicadores que compõem a variável desempenho organizacional, através do uso da análise de regressão, como ilustra a Tabela 1. Para tanto, foram executadas cinco análises de regressão múltipla separadamente, considerando-se os indicadores do desempenho organizacional como variáveis dependentes e as capacidades organizacionais como as variáveis independentes. 
Tabela 1 - Análise de regressão entre capacidades organizacionais e indicadores do desempenho organizacional

\begin{tabular}{|c|c|c|}
\hline $\begin{array}{l}\text { Capacidades Organizacionais/ } \\
\text { Desempenho Organizacional }\end{array}$ & $\begin{array}{l}\text { Coeficiente } \\
\text { Beta }\end{array}$ & Sig.* \\
\hline \multicolumn{3}{|l|}{ Taxa de ocupação } \\
\hline Clareza Estratégica & 0,165 & 0,046 \\
\hline \multicolumn{3}{|l|}{ Valor da diária média cobrada ao hóspede } \\
\hline Capital Intelectual & 0,197 & 0,017 \\
\hline \multicolumn{3}{|l|}{ Desempenho financeiro } \\
\hline Mudança & 0,247 & 0,003 \\
\hline \multicolumn{3}{|l|}{ Produtividade do trabalho } \\
\hline Capital Intelectual & 0,310 & 0,000 \\
\hline Mudança & 0,248 & 0,001 \\
\hline Eficiência Administrativa & 0,184 & 0,015 \\
\hline \multicolumn{3}{|l|}{ Qualidade dos serviços } \\
\hline Capital Intelectual & 0,406 & 0,000 \\
\hline Mudança & 0,257 & 0,000 \\
\hline Eficiência Administrativa & 0,207 & 0,004 \\
\hline
\end{tabular}

* Significância estatística considerada a 0,05 ou menos.

Observa-se que tanto a capacidade de Capital Intelectual quanto a capacidade de Mudança aparecem como determinantes de três dos cinco indicadores do desempenho organizacional, diferentemente das demais. Esse é um dos motivos pelos quais elas se sobressaíram no modelo estatístico desenvolvido. A capacidade de Capital Intelectual possui maiores pesos sobre a qualidade dos serviços e sobre a produtividade do trabalho, com coeficientes beta iguais a 0,406 e 0,310, respectivamente. Isso reforça a ideia de que o comprometimento dos funcionários e suas competências individuais contribuem diretamente com o resultado geral do trabalho, como sugerem os autores que seguem a linha da visão baseada em recursos (Lengnick-Hall et al., 2009; Mascarenhas, 2008; Wright, Dunford, \& Snell, 2001). Além disso, ela é a única capacidade que revelou influência sobre o valor da diária média cobrada ao hóspede. Já a capacidade de Mudança também interfere sobre a qualidade dos serviços e a produtividade do trabalho, além de ser a única que aparece relacionada diretamente ao desempenho financeiro, o que vem a corroborar a relevância da inovação e da rápida adaptação ao ambiente competitivo como um elemento determinante da continuidade e do aumento do desempenho organizacional (Ulrich, 2000).

A capacidade de Eficiência Administrativa exerce influência direta sobre apenas dois dos cinco indicadores do desempenho organizacional: qualidade dos serviços e produtividade do trabalho. Ambos são indicadores mais relacionados às atividades operacionais. A capacidade de Clareza Estratégica influencia diretamente a taxa de ocupação dos meios de hospedagem, que é um dos indicadores mais valorizados no setor hoteleiro, podendo-se considerar que ele depende fortemente das escolhas estratégicas realizadas pela organização, que vão impactar sobre o seu poder de atrair e fidelizar clientes. 
Essas análises proporcionadas pela técnica de regressão múltipla revelaram que todas as quatro capacidades organizacionais são determinantes de um ou mais indicadores do desempenho, o que vem a reforçar a hipótese de que todas elas deveriam estar relacionadas à variável desempenho organizacional, em maior ou menor intensidade.

Para averiguar se o modelo estatístico realmente poderia se sobressair e negar a hipótese debatida acima, foram criados, por meio da análise de conglomerados, dois grupos de meios de hospedagem. O primeiro grupo, composto por 63 hotéis, consegue desenvolver melhor as capacidades de Capital Intelectual e Mudança, sendo deficitários nas capacidades de Clareza Estratégica e Eficiência Administrativa. Logo, é um grupo mais caracterizado pela flexibilidade e voltado para as atividades de administração de pessoal. O segundo grupo, composto por 83 hotéis, é justamente o oposto, conseguindo desenvolver melhor as capacidades de Clareza Estratégica e Eficiência Administrativa, mas sendo fraco nas capacidades de Capital Intelectual e Mudança. É, pois, caracterizado pelo controle e voltado para as atividades de administração de processos.

Segundo o modelo estatístico apresentado na Figura 3, o grupo de meios de hospedagem que desenvolve melhor as capacidades de Capital Intelectual e Mudança deveria apresentar um desempenho organizacional superior, especialmente quando comparado ao segundo grupo. Contudo, o teste $\mathrm{t}$ de Student aplicado para comparar as médias não apontou diferenças estatisticamente significantes de desempenho organizacional entre os grupos analisados.

Ainda na tentativa de confirmar a hipótese do modelo teórico, foram criados outros dois grupos de meios de hospedagem. Nessa segunda análise, o primeiro grupo, formado por 57 hotéis, apresenta baixo nível de desenvolvimento de todas as quatro capacidades organizacionais, sendo denominado Equilíbrio Negativo. Em contraponto, o segundo grupo, formado por 90 hotéis, apresenta valores médios positivos de desenvolvimento das quatro capacidades organizacionais, o que significa que eles estão acima da média. Logo, recebeu o nome de Equilíbrio Positivo.

Aplicando-se o teste $t$ de Student para comparar as médias de desempenho organizacional desses dois grupos, uma diferença significativa foi encontrada, tendo o grupo Equilíbrio Positivo manifestado um desempenho superior ao grupo Equilíbrio Negativo. Essa constatação reitera a percepção de que é o desenvolvimento conjunto e equilibrado das quatro capacidades organizacionais que irá determinar o sucesso das organizações, conforme defendido por Ulrich (2000).

A partir das análises realizadas, pode-se argumentar que o desenvolvimento balanceado das capacidades organizacionais gera benefícios e resultados positivos para os meios de hospedagem que conseguem alcançar esse equilíbrio. Desse modo, os dados da pesquisa confirmam os pressupostos do Modelo de Múltiplos Papéis de Ulrich (1998; 2000). Vale lembrar que essa compreensão de equilíbrio também é uma premissa do Modelo de Valores Competitivos (Quinn \& Rohrbaugh, 1983; Cameron \& Quinn, 2006), que sugere que as quatro configurações de gestão de pessoas, apesar de parcialmente contraditórias, complementam-se. 


\section{CONSIDERAÇÕES FINAIS}

Diante dos resultados alcançados no estudo empírico, quanto às relações entre estratégias de gestão de pessoas, capacidades organizacionais e desempenho organizacional na hotelaria do Nordeste brasileiro, pôde-se chegar às conclusões descritas a seguir.

No que se refere à influência das estratégias de gestão de pessoas sobre as capacidades organizacionais do setor hoteleiro, as relações encontradas confirmam a sobreposição proposta por Cameron e Quinn (2006) entre as estratégias de gestão de pessoas do Modelo de Valores Competitivos e as capacidades organizacionais do Modelo de Múltiplos Papéis de Ulrich (1998) e revelam outras associações concomitantes e complementares.

Destarte, o desenvolvimento da capacidade de Clareza Estratégica requer a implementação de estratégias de gestão de pessoas com enfoque organizacional orientado para o ambiente externo, que promovam a busca por resultados e o alinhamento estratégico, aliados ao estímulo à transformação e ao empreendedorismo. O desenvolvimento da capacidade de Mudança também requer a implementação de estratégias de gestão de pessoas com foco no ambiente externo, voltadas para a inovação e melhoria contínua e para a produtividade e competitividade. O desenvolvimento da capacidade de Capital Intelectual demanda a implementação de estratégias de gestão de pessoas que promovam a satisfação das necessidades dos funcionários, a eficiência dos processos e a criatividade. $O$ desenvolvimento da capacidade de Eficiência Administrativa requer a implementação de estratégias de gestão de pessoas que evidenciem a melhoria contínua dos processos, o comprometimento e o trabalho em equipe, além do planejamento e da conquista de metas.

Essas múltiplas relações identificadas corroboram a perspectiva configuracional da gestão estratégica de pessoas ao sugerirem a existência de modelos híbridos, em que sejam contemplados elementos dos quatro modelos de gestão de pessoas aqui analisados, buscando uma combinação sinérgica entre eles para maximizar e usufruir dos benefícios que cada um é capaz de proporcionar.

Dentre as quatro capacidades organizacionais investigadas, apenas duas exercem influência direta sobre o desempenho organizacional: Mudança e Capital Intelectual, ambas voltadas para as atividades de administração de pessoal, apresentando maior ênfase sobre as pessoas e traduzindo maior flexibilidade. Esse resultado é coerente com a teoria da visão baseada em recursos, que advoga que as pessoas são os ativos mais valiosos das organizações e que representam uma fonte de vantagem competitiva sustentável (Barney, 1991), bem como com a teoria das capacidades dinâmicas, a qual sugere que o desenvolvimento de capacidades que favorecem a transformação e a adaptação ao ambiente também proporciona vantagens competitivas às organizações (Teece, Pisano, \& Shuen, 1997).

Apesar de existir suporte teórico para esse resultado, o Modelo de Múltiplos Papéis de Ulrich (2000) sugere uma ideia de equilíbrio entre as capacidades organizacionais, pois o autor defende que elas se complementam e devem ser desenvolvidas em conjunto para proporcionar 
diferencial competitivo às organizações. Essa noção de equilíbrio foi confirmada por análises complementares, que averiguaram que os hotéis que conseguem desenvolver as quatro capacidades organizacionais de forma conjunta e equilibrada alcançam um desempenho superior.

Diante do exposto, acredita-se que o estudo conseguiu ampliar a compreensão sobre as relações entre as estratégias de gestão de pessoas e o desempenho organizacional, inserindo as capacidades organizacionais como variável mediadora, contribuindo assim para destravar a "caixa preta" desse processo - como debatido na problemática deste trabalho.

Foi possível também comprovar as afirmações de Ulrich (2000), de que a gestão de pessoas desempenha a importante função de transformar estratégias em capacidades organizacionais. Estas, por sua vez, são resultados de gestão de pessoas críticos para o alcance de desempenho organizacional, promovendo o elo entre a gestão de pessoas e o desempenho.

Ao se tentar desvendar especificamente como esse processo ocorre, percebeu-se que tanto as configurações de estratégias de gestão de pessoas quanto as capacidades organizacionais são complementares e que dificilmente a escolha entre um ou outro modelo irá proporcionar um desempenho superior. Esse resultado confirma o teorizado por Legge (2005), de que não é uma simples questão de escolher entre modelos, mas sim de saber equilibrá-los na gestão de pessoas.

Desse modo, os resultados apresentados comprovaram que melhorias gerais no desempenho dos empreendimentos são efeito de um conjunto de práticas de gestão de pessoas interdependentes, que se reforçam mutuamente para a produção de efeito sinérgico. Logo, eles também contribuíram para subsidiar a compreensão da abordagem configuracional, que é a mais complexa entre as três abordagens teóricas da gestão estratégica de pessoas e a mais carente de suporte empírico.

Como sugestões para futuras pesquisas complementares, recomenda-se a condução de estudos similares em outras regiões geográficas do país, bem como em outros países, com características culturais distintas, para permitir comparações e identificar possíveis diferenças. Também se propõe o desenvolvimento de estudos qualitativos para esclarecer as relações aqui apresentadas e se aprofundar no processo de transformação das estratégias de gestão de pessoas em capacidades organizacionais e desempenho superior, proporcionando uma visão mais abrangente dos sistemas de gestão de pessoas e das práticas organizacionais. Recomenda-se, ainda, que futuras pesquisas testem a inserção de outras variáveis mediadoras na análise das relações entre estratégias de gestão de pessoas e desempenho organizacional, com o intuito de identificar fatores que possam, complementarmente às capacidades organizacionais, contribuir para a explicação da variação de desempenho das organizações.

\section{REFERÊNCIAS}

Albuquerque, L. G. (1999) Estratégias de recursos humanos e competitividade. In M. M. F. Vieira, \& L. M. B. Oliveira (Org.). Administração contemporânea: perspectivas estratégicas. São Paulo: Atlas.

Barney, J. (1991). Firm resources and sustained competitive advantage. Journal of Management, 17(1), pp. 
99-120.

Barney, J. B., \& Wright, P. M. (1998). On becoming a strategic partner: the role of human resources in gaining competitive advantage. Human Resource Management, 37(1), pp. 31-46.

Becker, B. E., Huselid, M. A., \& Ulrich, D. (2001). Gestão estratégica de pessoas com "scorecard": interligando pessoas, estratégia e performance. Rio de Janeiro: Elsevier.

Cameron, K. S., \& Quinn, R. E. (2006). Diagnosing and changing organizational culture. San Francisco: Jossey-Bass.

Deadrick, D. L., \& Gibson, P. A. (2009). Revisiting the research-practice gap in HR: a longitudinal analysis. Human Resource Management Review, 19(2), pp. 144-153.

Delery, J. E., \& Doty, D. H. (1996). Modes of theorizing in strategic human resource management: tests of universalistic, contingency and configurational performance predictions. Academy of Management Journal, 39( 4), pp. 802-835.

Jin, Y., Hopkins, M. M., \& Wittmer, J. L. S. (2010) Linking human capital to competitive advantages: flexibility in a manufacturing firm's supply chain. Human Resource Management, 49(5), pp. 939-963.

Ketkar, S., \& Sett, P. K. (2010). Environmental dynamism, human resource flexibility, and firm performance: analysis of a multi-level causal model. The International Journal of Human Resource Management, 21(8), pp. 1173-1206.

Lacombe, B. M. B.; Albuquerque, L. G. (2008). Avaliação e mensuração de resultados em gestão de pessoas: um estudo com as maiores empresas instaladas no Brasil. RAUSP. Revista de Administração, 43(1), pp. 5-16.

Legge, K. (2005). Human resources, realities and rethorics. London: Palgrave.

Lengnick-Hall, C. A., Beck, T. E., \& Lengnick-Hall, M. L. (2011). Developing a capacity for organizational resilience through strategic human resource management. Human Resource Management Review, 21(3),pp. 243-255.

Lengnick-Hall, M. L., Lengnick-Hall, C. A., Andrade, L. S., \& Drake, B. (2009). Strategic human resource management: the evolution of the field. Human Resource Management Review, 19(2), pp. 64-85.

Martín-Alcázar, F., Romero-Fernández, P. M., \& Sánchez-Gardey, G. (2008). Human resource management as a field of research. British Journal of Management, 19, pp.103-119.

Mascarenhas, A. O. (2008). Gestão estratégica de pessoas: evolução, teoria e crítica. São Paulo: Cengage Learning.

McClean, E., \& Collins, C. J. (2011). High-commitment HR practices, employee effort, and firm performance: investigating the effects of HR practices across employee groups within professional services firms. Human Resource Management, 50(3), pp. 341-363.

Miles, R. E., \& Snow, C. C. (1984). Designing Strategic Human Resource Systems. Organizational Dynamics, 13(1), pp. 36-52. 
Oliveira, P. M., Albuquerque, L. G., \& Muritiba, S. N. (2003). Uma análise da pesquisa sobre avaliação de resultados em gestão de pessoas na atualidade. Anais do Encontro da Associação Nacional de PósGraduação e Pesquisa em Administração, Atibaia, SP, Brasil, 27.

Panayotopoulou, L., Bourantas, D., \& Papalexandris, N. (2003). Strategic human resource management and its effects on firm performance: an implementation of the competing values framework. International Journal of Human Resource Management, 14(4), pp. 680-699.

Penrose, E. T. (1959). The theory of the growth of the firm. Oxford: Blackwell.

Quinn, R. E., \& Rohrbaugh, J. (1983). A spatial model of effectiveness criteria: towards a competing values approach to organizational analysis. Management Science, 29(3), pp. 363-377.

Roehling, M. V., Boswell, W. R., Caligiuri, P., Feldman, D., Graham, M. E., Guthrie, J. P., Morishima, M., \& Tansky, J. W. (2005). The future of HR management: research needs and directions. Human Resource Management, 44(2), pp. 207-216.

Schuler, R. S., \& Jackson, S. E. (1987). Linking competitive strategies with human resource management practices. The Academy of Management Executive, 1(3), pp. 207-219.

Schuler, R. S., \& Walker, J. W. (1990). Human resources strategy: focusing on issues and actions. Organizational Dynamics, 19(1), pp. 5-19.

Teece, D. J., Pisano, G., \& Shuen, A. (1997). Dynamic capabilities and strategic management. Strategic Management Journal, 18(7), pp. 509-533.

Truss, C., Gratton, L., Hope-Hailey, V., McGovern, P., \& Stiles, P. (1997). Soft and hard models of human resource management: a reappraisal. Journal of Management Studies, 34(1), pp. 53-73.

Ulrich, D. (1998). Os campeões de recursos humanos: inovando para obter os melhores resultados. São Paulo: Futura.

Ulrich, D. (Org.). (2000). Recursos humanos estratégicos: novas perspectivas para os profissionais de RH. São Paulo: Futura.

Ulrich, D., \& Smallwood, N. (2005). HR's new ROI: return on intangibles. Human Resource Management, 44(2), pp. 137-142.

Wei, L., \& Lau, C. (2010). High performance work systems and performance: the role of adaptive capability. Human Relations, 63(10), pp. 1487-1511.

Wright, P. M., Dunford, B. B., \& Snell, S. A. (2001). Human resources and the resource based view of the firm. Journal of Management, 27, pp. 701-721.

Wright, P. M., McMahan, G. C., \& McWilliams, A. (1994). Human resources and sustained competitive advantage: a resource-based perspective. The International Journal of Human Resource Management, 5(2), pp. 301-326. 